\title{
MÉXICO ANTE EL FUTURO DE APEC
}

CARlos UsCanga Prieto

ORCID: https://orcid.org/0000-0002-2010-227X

Universidad Nacional Autónoma de México

\section{INTRODUCCIÓN}

Dos décadas y media marcaron en 2019 el ingreso de México en el foro de Cooperación Económica Asia Pacífico (APEC), tiempo durante el cual ha participado con diferentes intensidades y compromisos. En este sentido, obliga a realizar un ejercicio retrospectivo de cómo la representación mexicana en ese mecanismo regional se ha desempeñado, remarcando la naturaleza de su estrategia y alcances en su despliegue. Lo anterior es una condicionante para, también, poder delinear cómo México identifica al APEC en el futuro y, en particular, cómo el gobierno mexicano se ve reflejado en el mismo para el cumplimiento de sus metas e intereses nacionales en el ámbito regional y multilateral.

A tal efecto, el presente artículo parte de la exploración de los diferentes periodos que han marcado la inserción mexicana en ese espacio de diálogo intergubernamental, para después hacer un ejercicio prospectivo a fin de trazar algunos escenarios basados en tres supuestos: el papel del ejecutivo mexicano en la agenda internacional, el estado de la energía institucional del Estado mexicano para enfrentar los retos estructurales y coyunturales que implica la agenda internacional, y por último, los compromisos particulares hacia el APEC en términos de atender la llamada "crisis del multilateralismo" motivada por las políticas proteccionistas de los Estados Unidos bajo la presidencia de Donald Trump, así como los

D.R. ( ) 2020. Anuario Asia Pacífico Licencia Creative Commons Atribución-NoComercial-SinDerivar (CC BY-NC-ND) 4.0 Internacional 
efectos de la cancelación por parte de Chile de la reunión ministerial y Cumbre Informal de Líderes en 2019.

Asimismo, se parte del supuesto de que México está anclado dentro de la profunda integración con la economía estadounidense, en la que el Tratado de Libre Comercio de América del Norte (TLCAN) y el Tratado México-Estados Unidos-Canadá (T-MEC) son una expresión más que marca las pautas de la política comercial externa mexicana y su interacción con otras regiones del mundo, además de su posicionamiento en los foros regionales de cooperación. Lo anterior se aprecia en APEC como una posición de cautela, y muy cuidadosa, para no abanderar elementos que pudieran causar desencuentros con Washington, por lo que su presencia en ese foro regional es de bajo perfil pero con capacidad de reacción a los temas de la agenda, desplegando destellos de activismo, pero no de un liderazgo integral.

\section{LA TRAZABILIDAD DE MÉXICO EN APEC}

Después de un poco más de un cuarto de siglo de permanencia de México en APEC, es necesario analizar la relevancia que ese mecanismo regional ha tenido para la política comercial de México. En una primera apreciación parecería que la simple decisión de obtener su membresía, en 1993, reflejaba (casi de manera automática) el deseo del gobierno mexicano en considerarlo un elemento central en sus estrategias de inserción dentro de la Cuenca del Pacífico.

Sin duda, la adhesión a ese foro se consideraba un paso relevante, por lo que puede plantearse la siguiente pregunta: ¿cuál ha sido el papel que nuestro país ha tenido en el APEC? Para poder responder es importante realizar un ejercicio de trazabilidad que permita valorar su desempeño dentro del foro para saber con mayor precisión cómo México ha tenido capacidad de respuesta, pero sobre todo de incidencia en su trayectoria, que - en una apreciación preliminar — no ha sido contundentemente proactiva si se toma como indicador que sólo en una ocasión ha organizado la Cumbre Informal de Líderes, en la que, como sede, puede delinear temas centrales para su discusión entre los miembros y contribuir al desarrollo de los trabajos de ese foro regional. 
Entonces ¿por qué México no ha desplegado acciones y estrategias para un protagonismo en APEC? Lo anterior podría explicarse por dos elementos: por un lado, la pertenencia al TLCAN y la creciente integración al mercado de los Estados Unidos hicieron que la política comercial mexicana fuera muy cautelosa y de "autocontención" para no generar escenarios de desacuerdos frente a su primer socio comercial. Lo anterior se suma al argumento de José León-Manríquez y de Luis F. Álvarez (2020): la gran sombra del TLCAN ha generado una estrategia de integración pasiva hacia la región del Pacífico asiático. Por otro lado, si bien los funcionarios directamente responsables de coordinar y atender la agenda de México en APEC han tenido continuidad (a pesar de los cambios del partido en el poder), es posible identificar que no ha existido - de manera manifiesta y contundente - una alta prioridad en los tomadores de decisión de más alto nivel para colocar los temas de APEC como centrales en la política exterior económica más allá de declarar las "potenciales oportunidades" que ofrece el Pacífico asiático y de las intenciones - reiteradamente manifiestas - en avanzar en la diversificación económica y de sus mercados de exportación (Secofi, 1997-1998). No obstante, ha existido amplia capacidad para atender los temas de ese mecanismo regional, en particular en la facilitación del comercio e inversión, cooperación económica y técnica, y en dar respuesta, en los márgenes posibles, a las metas de liberalización económica establecidas en Bogor en 1994.

En ese contexto, para efectos del presente análisis, pueden identificarse tres etapas. La primera (Ingreso y aprendizaje) parte de su incorporación hasta el 2001 cuando inicia el giro de ese mecanismo, al incluir, por presiones de Washington, la agenda de seguridad como resultado de los ataques terroristas en las Torres Gemelas y el Pentágono. La incorporación de México en el APEC se logró en 1993 en la reunión de Blake Island (formalizada un año después), el consenso entre los miembros estuvo condicionado por los países del ASEAN para que también se aceptara el ingreso de Papúa Nueva Guinea. Es un hecho conocido el decisivo apoyo de Washington, ya que era necesario que México, en el marco de las negociaciones del TLCAN, estuviera representado en ese nuevo organismo intergubernamental de carácter transpacífico.

Las aspiraciones del gobierno mexicano estaban ligadas a que el ingreso en el APEC favorecería la cimentación del modelo neoliberal a través del fortalecimiento de la recomposición económica basada en los principios de apertura comercial, la desregulación 
de su economía y la ampliación de sus mercados de exportación (Cárdenas, 1993). Sin embargo, la "diplomacia de la pertenencia" (Uscanga, 2000a), cuyo fin era el ingreso, se convirtió en una meta en sí misma, sin tener una estrategia clara de lo que implicaba su participación en ese nuevo mecanismo de cooperación. El error de diciembre 1994 marcó el discurso de México acerca de la recuperación económica, y cuando estalló la crisis asiática en 1997 se consideró necesario compartir su experiencia con las economías asiáticas, las cuales expresaron diversas reacciones al respecto (Uscanga, 2000b). A tal efecto, el gobierno de Ernesto Zedillo se limitó a dar curso a las discusiones dentro del nuevo foro regional, sin el despliegue de una estrategia diplomática más asertiva, considerándolo un espacio multilateral más adonde podía asistir el ejecutivo mexicano (Anguiano, 2001).

No obstante, el foro era una plataforma que, al menos en esta etapa, complementaba las estrategias de gobierno. Las políticas de ajuste económico eran compatibles con las agendas de liberalización económica en el marco de APEC, y México presentó sus Planes de Acción Individual (PAI o IAP, por sus siglas en inglés), que indicaban acciones ya implementadas en el marco del "consenso de Washington" en materia de liberalización, desregulación y apertura comercial. Al gobierno mexicano le costó mucho entender los principios "consenso", "acciones voluntarias" y "compromisos no vinculantes", que eran parte de la filosofía de funcionamiento de APEC y estaban ligados a las constantes críticas que Estados Unidos hacía de manera recurrente, apuntando la necesidad de la estricta "reciprocidad" para el avance de los procesos de apertura comercial y financiera.

Asimismo, México consideraba, en lo general, que las metas de Bogor que marcaban una liberalización económica diferenciada (para las economías en desarrollo en 2010 y 2020) eran viables, aunque no representaban una fórmula única para lograr esos compromisos. Asimismo, se pronunciaba en favor de apoyar a la OMC para avanzar en la agenda en materia de comercio e inversión, pero también en profundizar la cooperación económica y técnica en el marco del llamado ECOTECH (véase cuadro 1).

Cuadro 1. México en APEC, 1996-2000

\begin{tabular}{|l|l|l|l|}
\hline Año & Economía sede & \multicolumn{1}{|c|}{ Acciones } & \multicolumn{1}{c|}{ Participación } \\
\hline 1996 & Filipinas & $\begin{array}{l}\text { - Presenta Plan de Acción Individual } \\
\text { (PAI) que incluye medidas de } \\
\text { liberalización de la inversión }\end{array}$ & $\begin{array}{l}\text { - Cuarta Cumbre de Líderes en } \\
\text { Manila, Filipinas (Ernesto Zedillo } \\
\text { Ponce de León) }\end{array}$ \\
\hline
\end{tabular}




\begin{tabular}{|c|c|c|c|}
\hline & & $\begin{array}{l}\text { extranjera, simplificación de } \\
\text { procedimientos aduaneros, } \\
\text { desregulación de la actividad } \\
\text { económica y derechos de propiedad } \\
\text { intelectual } \\
\text { - Se pronuncia para apoyar la } \\
\text { cooperación económica y técnica } \\
\text { - Involucrar a los empresarios en } \\
\text { APEC }\end{array}$ & $\begin{array}{l}\text { - Reuniones ministeriales } \\
\text { - } \text { SOM } \\
\text { - } \text { CTI (Comité de Comercio e } \\
\text { Inversión) } \\
\text { - Grupo de trabajo y de expertos }\end{array}$ \\
\hline 1997 & Canadá & $\begin{array}{l}\text { - Presenta nueva versión de su PAI } \\
\text { - Se opone al enfoque de } \\
\text { liberalización sectorial anticipada } \\
\text { - Apoyo a APEC para que impulse una } \\
\text { liberalización comprehensiva a } \\
\text { través de la OMC }\end{array}$ & $\begin{array}{l}\text { - Quinta Cumbre de Líderes en } \\
\text { Vancouver, Canadá (Ernesto Zedillo } \\
\text { Ponce de León) } \\
\text { - Reuniones ministeriales } \\
\text { - SOM } \\
\text { - CTI } \\
\text { - Grupo de trabajo y de expertos }\end{array}$ \\
\hline 1998 & Kuala Lumpur & $\begin{array}{l}\text { - Presenta versión mejorada de PAI } \\
\text { - Apoya los tiempos de liberalización } \\
\text { económica en el marco de las metas } \\
\text { de Bogor } \\
\text { - Liberalización comprehensiva }\end{array}$ & $\begin{array}{l}\text { - Sexta Cumbre de Líderes en Kuala } \\
\text { Lumpur, Malasia (Ernesto Zedillo } \\
\text { Ponce de Léon) } \\
\text { - Reuniones ministeriales } \\
\text { - SOM } \\
\text { - CTI } \\
\text { - Grupo de trabajo y de expertos }\end{array}$ \\
\hline 1999 & Nueva Zelanda & $\begin{array}{l}\text { - Presenta más avances PAI } \\
\text { - Metas de Bogor como medio para la } \\
\text { liberalización económica, pero no } \\
\text { excluye otras vías } \\
\text { - Apoyo a la liberalización } \\
\text { comprehensiva y nueva ronda de } \\
\text { negociaciones multilaterales en la } \\
\text { OMC } \\
\text { - México como sede de APEC en } 2002\end{array}$ & $\begin{array}{l}\text { - Séptima Cumbre de Líderes en } \\
\text { Auckland, Nueva Zelanda (Ernesto } \\
\text { Zedillo Ponce de León) } \\
\text { - Reuniones ministeriales } \\
\text { - SOM } \\
\text { - CTI } \\
\text { - Grupo de trabajo y de expertos }\end{array}$ \\
\hline
\end{tabular}

FuENTE: Secretaría de Comercio y Fomento Industrial (1996-2000).

En esta primera etapa, indudablemente, la diplomacia económica mexicana visualizó Asia Pacífico a través de la participación en APEC como una herramienta útil para proyectar y hacer más visible a México en la región. De manera independiente a los resultados obtenidos, es cierto que el gobierno tuvo que desplegar acciones más puntuales para atender la agenda de APEC, cuyas dependencias asistieron a las múltiples reuniones en los diferentes comités y grupos de trabajo. Los funcionarios de Secofi y SRE se reunían con sus pares de manera más frecuente y, sobre todo, ese espacio regional podía representar una plataforma para atender asuntos bilaterales en el más alto nivel en el marco de la Cumbre Informal de Líderes y en los encuentros ministeriales (Ojeda, 2000). En suma, la atención de los temas de Asia y el Pacífico se hizo notoria en la política exterior mexicana, lo cual generaba 
diferentes niveles de respuesta. Asimismo, el ejecutivo mexicano se apropiaba del discurso sobre APEC, ya que al menos una vez al año se debía reunir con sus máximos representantes de las otras economías.

La política exterior económica mexicana, en lo general, se mantuvo con la llegada del Partido Acción Nacional (PAN) al poder en la presidencia de Vicente Fox Quesada. La agenda comercial en Asia Pacífico se centró en APEC y en la firma limitada de instrumentos de libre comercio en el marco de la moratoria declarada para la negociación de nuevos TLC (Uscanga, 2004). A pesar de lo anterior, México negoció con Japón un Acuerdo de Asociación Económica (AAE) que entró en vigor en 2005 siendo el único hasta la fecha que —en términos bilaterales - tiene activo con un país asiático. Para principios del nuevo siglo, se observaba un cambio de las expectativas que APEC podía ofrecer para sus miembros. La construcción de consensos se percibía compleja, y al cumplimiento de las metas de Bogor se sumaban más obstáculos (para las economías que debían aplicar el modelo de reciprocidad y acuerdos vinculantes) a través de las acciones voluntarias. También se valoraba que, a pesar de las discusiones en los comités y grupos de trabajo, así como en los proyectos en el marco de ECOTECH, no había generado resultados más específicos y concretos. Asimismo, se veía que APEC, si bien era un espacio de diálogo regional relevante, no tenía la fuerza y capacidad suficientes (como se demostró en la crisis asiática de 1997) para ser referente respecto a los problemas globales.

La segunda etapa (Estrategia selectiva y focalizada) está marcada por la participación mexicana dentro de APEC en medio de las citadas críticas y la búsqueda de cambios en su funcionamiento. Las grandes expectativas de su creación se transformaban en deseo de "reencauzar" sus trabajos. Ese contexto lo marcaron los preparativos iniciados en 2001 para celebrar la reunión ministerial, la de altos funcionarios (SOM) y la Cumbre informal de Líderes en 2002, en la que el gobierno mexicano presentó temas y dio seguimiento a una agenda con diversos grados de participación y "liderazgo orientado a tareas" reflejado en los grupos de trabajo, estrategia que continuó hasta 2018. Es decir, México adoptó una posición conservadora y no confrontacional en aspectos sensibles, seleccionando sus áreas de acción en tópicos específicos y de corte técnico, evitando propuestas protagónicas al interior de las problemáticas centrales de APEC, pero sí adhiriéndose a algunas en el nivel declarativo. 
Como es sabido, la introducción de temas de seguridad definió la Cumbre informal de Líderes en China en 2001 (la primera a la que asistió Vicente Fox Quesada) y la lucha contra el terrorismo en la agenda de APEC. Las vicisitudes de la posición mexicana para apoyar a los Estados Unidos en su cruzada contra Irak en el marco del Consejo de Seguridad de la Organización de las Naciones Unidas (ONU) fueron elementos que convergieron en la organización, por parte de México, de la reunión ministerial y la Cumbre de Líderes en 2002 bajo la responsabilidad de la Secretaría de Economía (el 25 de junio de 2001 se creó el comité organizador de APEC), que había sustituido a su antecesora Secofi (Traslosheros, 2019). En medio de ese clima de tensión, el gobierno mexicano impulsó al tema eje sobre la "expansión de los beneficios del crecimiento y el desarrollo económico" entre los miembros de ese foro, marcando prioridades en forjar capacidades; y como tópicos particulares las relaciones entre las micro, pequeñas y medianas empresas, la profundización de la facilitación del comercio, el papel de los jóvenes y las mujeres en la llamada nueva economía (Derbez, 2002).

Asimismo, en la reunión de Los Cabos en 2002 hubo pronunciamientos en torno a que las acciones terroristas no fueran obstáculo para el comercio y los flujos de inversión, así como el respaldo de las negociaciones multilaterales en el marco de la OMC, en su misión para fomentar un comercio más abierto, eliminando las barreras como los subsidios agrícolas, las prácticas antidumping y cuotas compensatorias (Secretaría de Economía, 2003).

México, después de la organización de 2002, adoptó, por medio de la Secretaría de Economía, una estrategia de atención y coordinación de los temas de la creciente agenda múltiple de ese foro regional. Esa dependencia expresaría en su informe anual de labores que “en los años sucesivos a su presidencia, México ha continuado impulsando los temas y compromisos en 2002, colaborando en la definición de iniciativas y mejores prácticas que contribuyan a intensificar [las] relaciones económicas y cooperación con la región Asia Pacífico" (Secretaría de Economía, 2006, p. 62). Lo anterior implicaba también dar seguimiento a lo acordado en Los Cabos pero también adherirse o reaccionar en otros aspectos abordados en APEC (véase cuadro 2). 
CUADro 2. México en APEC, 2003-2006

\begin{tabular}{|c|c|c|c|}
\hline Año & Economía sede & Acciones & Cumbre de Lideres \\
\hline 2003 & Tailandia & $\begin{array}{l}\text { - Avances en la simplificación aduanera } \\
\text { - Desarrollo de la economía digital } \\
\text { - La lucha contra el terrorismo no debe mermar la } \\
\text { facilitación del comercio e inversión }\end{array}$ & Vicente Fox Quesada \\
\hline 2004 & Chile & $\begin{array}{l}\text { - Impulso político para el avance de las } \\
\text { negociaciones en la OMC }\end{array}$ & Vicente Fox Quesada \\
\hline 2005 & Corea del Sur & $\begin{array}{l}\text { - Evaluación de las Metas de Bogor } \\
\text { - Apoyo al libre comercio }\end{array}$ & Vicente Fox Quesada \\
\hline 2006 & Vietnam & $\begin{array}{l}\text { - Reforma de APEC como institución } \\
\text { - Fortalecer capacidades en el desarrollo de recursos } \\
\text { humanos } \\
\text { - Apoyo de PyMEs } \\
\text { - Impulso comercio e Inversión }\end{array}$ & $\begin{array}{l}\text { Ausencia del ejecutivo } \\
\text { mexicano (el Legislativo } \\
\text { no dio permiso de } \\
\text { ausentarse del país) }\end{array}$ \\
\hline
\end{tabular}

FUENTE: Secretaría de Economía (2003-2006).

Al final del sexenio de Vicente Fox, el Legislativo negó el permiso al presidente para ausentarse del país, por lo que éste no acudió a la Cumbre Informal de Líderes en Vietnam, siendo la primera ocasión en que el responsable del gobierno mexicano estuvo ausente. Ya en la administración de Felipe Calderón Hinojosa, en lo general, se mantuvo el seguimiento de los temas de APEC como el referido a la necesidad de profundizar los procesos de integración económica regional (Secretaría de Economía, 2006). En 2008 presentó el segundo examen de revisión de su PAI, y en noviembre de ese mismo año se implementó la tarjeta de viajes para hombres de negocios de APEC. En 2011 el presidente mexicano canceló su viaje a la XIX Cumbre de Líderes de APEC en Hawái, los Estados Unidos, por el accidente aéreo que produjo la muerte del secretario de Gobernación, José Francisco Blake Mora, siendo la segunda ocasión que el ejecutivo mexicano no estuvo presente en APEC.

Como se mencionó, era claro que en el inicio de la segunda década del siglo XXI, México compartía, junto a otros miembros, la opinión de que el perfil institucional de APEC ya no favorecía las expectativas creadas en ese foro regional. Para muchas economías participantes, la carencia de energía institucional estaba en sus propios principios fundacionales y la falta de acuerdos vinculantes, lo que desestimaba una real voluntad política para avanzar pronto en la agenda de liberalización, ya que había la percepción de que la apertura de los mercados de manera unilateral sería altamente desfavorable. Asimismo, se 
enfrentaba al hecho de que la liberalización económica no pudo emprender el camino trazado en las metas de Bogor, por lo que surgían otras opciones para ampliar la integración de las economías de ese foro regional. Asimismo, APEC sumaba otros puntos de discusión en su agenda como el tema de su reforma, y tópicos globales como cambio climático, crecimiento sostenible y sustentable.

En ese sentido, es interesante observar cómo APEC ha sido una caja de resonancia regional ante los temas de la agenda global. Para María Cristina Hernández y Jason Martínez Jurado (2019), México se ha adherido tradicionalmente a los pronunciamientos de rechazo a las prácticas proteccionistas, y ha expresado, en diferentes momentos, el apoyo a la OMC como garante de un sistema abierto multilateral de comercio. Asimismo, el cumplimiento de las diferentes hojas de ruta en diferentes sectores desde la competitividad, género, reforma estructural hasta los aspectos de crecimiento incluyente, conectividad física y digital. En efecto, el gobierno mexicano en las reuniones ministeriales y en el SOM ha reiterado la importancia de la OMC como centro de la gobernanza comercial a nivel mundial y ser un espacio promotor de diálogo entre sus miembros (Secretaría de Economía, 2018a).

Así, los autores citados consideran que la mejor manera de valorar la participación de México en el APEC debe ubicarse en tres aspectos: en primer lugar, en la participación mexicana en las reuniones, donde se puede apreciar su posicionamiento, marcando sus prioridades de incidencia en temas propios a ese foro, así como en temas multilaterales (véase cuadro 3).

Es interesante identificar la estrategia mexicana (como también lo hacen otras economías) de alinear las acciones internas de carácter institucional con los temas de APEC. Durante la gestión de Vicente Fox Quesada se elevó el tema de las PyMEs, y durante la administración de Enrique Peña Nieto el tema de las reformas estructurales. Esas acciones de convergencia son deseables, pero la estrategia de focalizar y seleccionar también implicaba que, en otros rubros de la agenda, las aportaciones mexicanas pudieran calificarse como incidencias "microtemáticas", sumándose simplemente a la discusión de los aspectos centrales de APEC, como el avance de la integración económica regional, las metas de Bogor, el Acuerdo de Libre Comercial de Asia Pacífico (ALACP o FTAAP por sus siglas en inglés) e incluso la agenda Post 2020. 
CUADRO 3. Prioridades temáticas de México en APEC (2013-2018)

\begin{tabular}{|c|c|c|c|c|}
\hline Año & Economía sede & Temas APEC & Temas globales & Cumbre de Lideres \\
\hline 2013 & Indonesia & $\begin{array}{l}\text { - Inversión en infraestructura } \\
\text { - Capital humano } \\
\text { - Difundir las reformas } \\
\text { - Mejorar la conectividad en todos } \\
\text { los niveles }\end{array}$ & & Enrique Peña Nieto \\
\hline 2014 & China & $\begin{array}{l}\text { - Apoyar la apertura económica } \\
\text { - Avanzar en la integración regional } \\
\text { - Mejora de la infraestructura } \\
\text { - Simplificación para la movilidad } \\
\text { de las personas }\end{array}$ & & Enrique Peña Nieto \\
\hline 2015 & Filipinas & $\begin{array}{l}\text { - Participar en el segundo plan para } \\
\text { la facilidad de negocios } \\
\text { - Apoyar las acciones de APEC } \\
\text { sobre reforma estructural } \\
\text { - Profundizar la integración } \\
\text { económica regional } \\
\text { - Fomentar la participación de } \\
\text { PyMEs } \\
\text { - Desarrollo de capital humano } \\
\text { construyendo comunidades } \\
\text { sostenibles y resilientes } \\
\text { - Mejora del comercio de servicios } \\
\text { Fortalecimiento de mercados } \\
\text { financieros }\end{array}$ & & Enrique Peña Nieto \\
\hline 2016 & Perú & $\begin{array}{l}\text { - Apoyar un eventual FTAAP } \\
\text { - Contribuir para la consecución de } \\
\text { las Metas de Bogor } \\
\text { - Apoyar el crecimiento incluyente } \\
\text { - Reforzar las acciones para la } \\
\text { facilitación de comercio } \\
\text { - Apoyar inserción de las PyMEs } \\
\text { - Apoyar a las mujeres en las } \\
\text { economías APEC }\end{array}$ & $\begin{array}{l}\text { - Enfrentar los retos } \\
\text { del cambio climático } \\
\text { - Seguridad } \\
\text { alimentaria }\end{array}$ & Enrique Peña Nieto \\
\hline 2017 & Vietnam & $\begin{array}{l}\text { - Reforma estructural } \\
\text { - Capacidades en recursos humanos } \\
\text { en la era digital } \\
\text { - Inclusión económica, financiera y } \\
\text { social } \\
\text { - Seguimiento de la estrategia de } \\
\text { APEC sobre educación } \\
\text { - Metas de Bogor sobre comercio e } \\
\text { inversión } \\
\text { - Mayor participación de PyMEs en } \\
\text { las cadenas globales }\end{array}$ & $\begin{array}{l}\text { Fortalecer el sistema } \\
\text { multilateral de } \\
\text { comercio } \\
\text { - Impulsar agenda } \\
2030\end{array}$ & Enrique Peña Nieto \\
\hline 2018 & $\begin{array}{l}\text { Papúa Nueva } \\
\text { Guinea }\end{array}$ & $\begin{array}{l}\text { - Colaborar en acciones } \\
\text { individuales y colectivas para } \\
\text { lograr las Metas de Bogor } \\
\text { - Apoyar Grupo de Visión para la } \\
\text { Agenda Post } 2020\end{array}$ & & $\begin{array}{l}\text { Ausencia del } \\
\text { ejecutivo mexicano } \\
\text { (que se sumó a la } \\
\text { inasistencia de } \\
\text { Donald Trump, }\end{array}$ \\
\hline
\end{tabular}




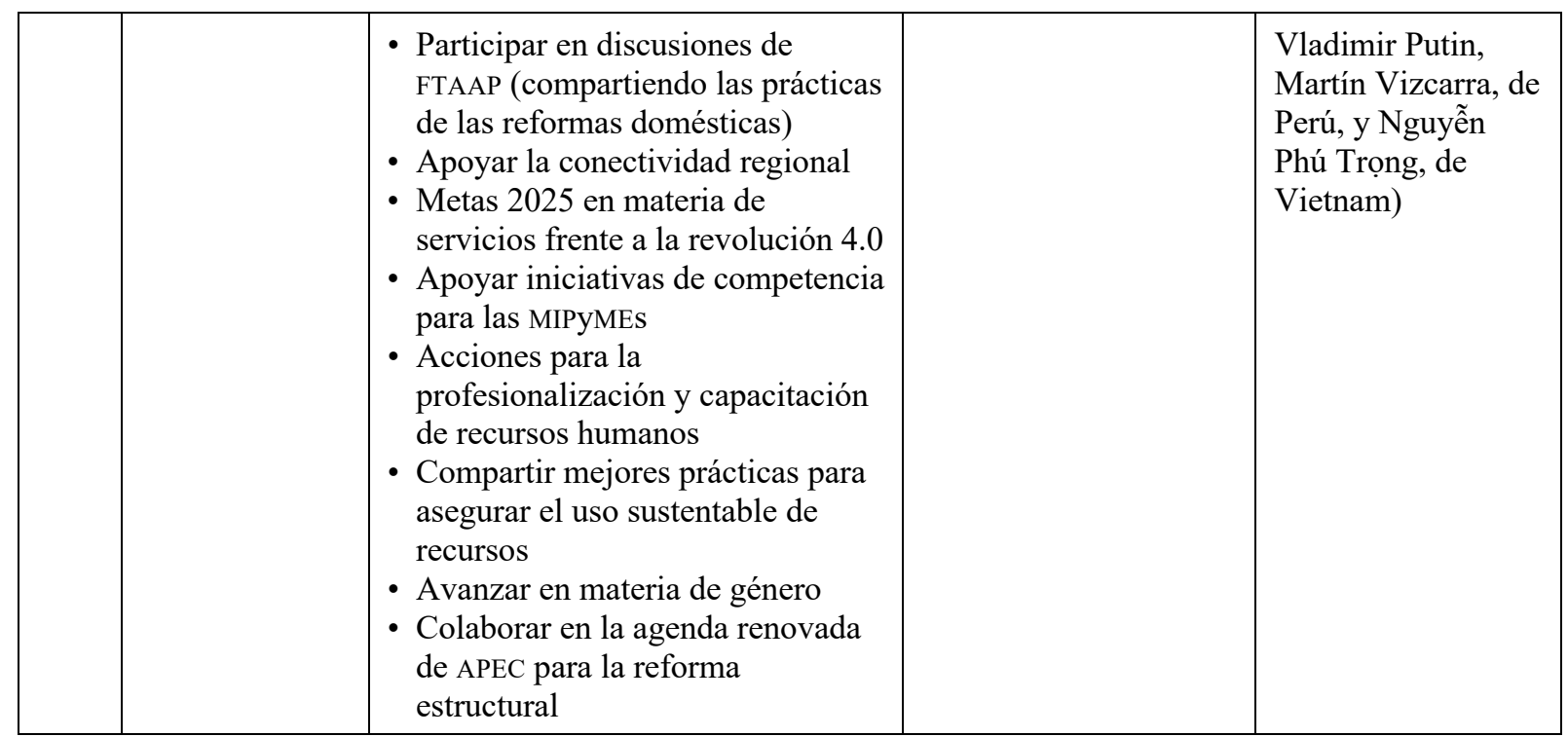

FUENTE: Secretaría de Economía (2018b).

En segundo lugar, los autores consideran que una métrica útil es la valoración de las propuestas e iniciativas de México dentro de APEC, cuyo liderazgo puede reflejarse en los grupos de trabajo; y en tercero, al interior de las actividades en la cooperación económica y técnica (ECOTECH). En ese contexto, México ha presidido varios grupos al interior de APEC, como el de Propiedad Intelectual (2010-2018), bajo el Instituto Mexicano de Propiedad Intelectual (IMPI); turismo (2011-2015), a cargo de la Secretaría de Turismo, y en el grupo de amigos de Reforma Regulatoria (2016 a la fecha), teniendo como responsable a la Comisión Federal de Mejora Regulatoria (Cofemar). Además de otras áreas en las que ha aprovechado las actividades de cooperación técnica para la formación de capacidades con fondos APEC. Entre 2009 y 2017 se beneficiaron cerca de 700 funcionarios mexicanos en temas de competencia, reforma estructural, servicios, inversión, propiedad intelectual, entre los más importantes (Secretaría de Economía, 2018b).

De acuerdo con el libro blanco sobre Política Comercial Internacional, los proyectos implementados por el gobierno mexicano con financiamiento de APEC se incrementaron de dos en el 2014 a 10 en el 2018, teniendo un total acumulado durante ese periodo de 21 . Si bien lo anterior es significativo, como puede observarse, México volcó su estrategia selectiva y focalizada para proyectar su visibilidad en temáticas más concentradas en la facilitación del comercio e inversión e incrementar significativamente su participación en los proyectos de cooperación. 
La tercera etapa general (Continuidad y ajustes en la austeridad) inició con la llegada de Andrés Manuel López Obrador mediante la implementación de una estrategia internacional concentrada en los Estados Unidos, con lo cual se ha ocasionado una merma en el discurso oficial del Ejecutivo en su aproximación a otras regiones del mundo. Lo anterior está relacionado con la política de austeridad (en diferentes momentos implementada en la administración pública federal en sexenios anteriores), y es claro que ahora ha erosionado con mayor profundidad la base instrumental de la política comercial, otrora la joya de la corona de la política exterior de México.

No obstante, en los temas de APEC no se visualizan — al menos en el corto plazocambios sustanciales, por lo que se mantiene el posicionamiento de México de focalizar y seleccionar pero con menos recursos institucionales; y, sobre todo, con la carencia de la apropiación del tema por parte del Ejecutivo mexicano ante un entorno económico internacional volátil, y con la erosión del papel de los organismos regionales y multilaterales, y la oleada del proteccionismo y la emergencia de tensiones comerciales entre los Estados Unidos y China, así como de Japón con Corea del Sur dentro del este de Asia.

En efecto, es indudable que después de tres décadas del nacimiento de APEC, las expectativas de su importancia y peso dentro de las políticas comerciales de las economías que la integran han cambiado de manera sustancial, en comparación con 1989, cuando había un deseo de que ese nuevo foro profundizara la cooperación para eliminar los obstáculos del comercio e inversión, y posteriormente servir de plataforma regional para enfrentar el proteccionismo y favorecer el libre comercio ante el agotamiento de la ronda de Uruguay del entonces Acuerdo General sobre Aranceles Aduaneros y Comercio (GATT). Hoy día el proteccionismo ha revestido nuevas formas, las cuales se han acendrado ante las tensiones comerciales impulsadas por las políticas de Donald Trump. Lo anterior ha generado la denominada crisis del multilateralismo, cuya señal fue la retirada de los Estados Unidos del Acuerdo de Asociación Transpacífico (TPP), como una de las primeras acciones ejecutivas del presidente estadounidense en 2017 , siendo un primer entregable a sus lectores con base en su política America First (Uscanga, 2018).

El esfuerzo de Japón, México y las economías restantes por salvar el valor acumulado de la negociación del TPP y la llegada final del consenso para avanzar en el TPP-11 (TPP sin 
los Estados Unidos), que después se nombraría СРTPP (Comprehensive and Progresive Transpacific Partnership), no fueron suficiente para atenuar las demandas de Washington para la firma de acuerdos de comercio de tipo bilateral. México y Canadá fueron las primeras víctimas del retorno de la "unilateralidad" en materia comercial de los Estados Unidos (De la Mora Sánchez, 2019).

En ese contexto, la política comercial de México se concentró en las negociaciones trilaterales para la renovación del Tratado de Libre Comercio de América del Norte (TLCAN), en cuya negociación final tuvo que ceder para modificar el contenido de las reglas de origen, entre otros aspectos importantes. En el plano interno en México, la derrota del Partido Revolucionario Institucional (PRI) en las elecciones de junio de 2018 y la llegada al poder de Andrés Manuel López Obrador influyeron directamente en poner dentro de un segundo plano la agenda Asia Pacífico, a pesar de la insistencia de los socios del este de Asia, en particular de China, Corea del Sur y Japón para hacer consultas con el gobierno saliente de Enrique Peña Nieto sobre los impactos que tendría el Tratado México-Estados Unidos-Canadá (TMEC).

Asimismo, los temas de APEC dejaron de ser visibles (en términos del discurso oficial), y como evidencia quedó la decisión del presidente Peña Nieto de no asistir a la Cumbre informal de líderes en Papúa Nueva Guinea, lo que significó la tercera ocasión en que México no estaba representado por el Ejecutivo en ese foro regional. Con ello, sumando la cancelación de la reunión de APEC en Chile en 2019, la gestión y sobre todo la reacción mexicanas (de perfil pragmático) continuarán atendiendo los compromisos derivados de la agenda acumulada y las obligaciones agregadas, en una clara merma en los identificables astibos de activismo desarrollados en la participación mexicana en los comités y grupos de trabajo.

Es un hecho, como ya se ha esbozado, que la agenda de APEC ha crecido de manera exponencial en temas ligados al propio foro regional, pero con otros aspectos que, de manera transversal, cubren aspectos multitemáticos, que se atienden en los diversos comités y grupos de trabajo en su interior. De la Cumbre de Líderes en Port Moresby a los trabajos realizados para la preparación de la malograda reunión en Santiago de Chile, los vectores giraron alrededor de cinco grandes rubros: los esfuerzos para profundizar la Integración Económica 
Regional (REI, por sus siglas en inglés), la mejora de la conectividad, el crecimiento incluyente y sustentable, la reforma estructural, la visión Post-2020 y el apoyo al sistema de comercio mundial libre y abierto.

Sobre ese último tema en particular, las posiciones de México en Papúa Nueva Guinea 2018 pueden concentrarse en cinco rubros: $a$ ) apoyo político de los Líderes de APEC para la mejora del funcionamiento de la OMC; $b$ ) continuar los trabajos hacia las Metas de Bogor en áreas identificadas; c) mantener la unión de los miembros en lograr el objetivo de un comercio libre y abierto a través del FTAAP; $d$ ) refrendar el compromiso hacia el sistema multilateral de comercio con los esfuerzos de integración como el CPTPP, y e) adoptar el pragmatismo y flexibilidad como un medio para la construcción de consensos entre los miembros de APEC y para atender los compromisos con el libre comercio y enfrentar el proteccionismo (Secretaría de Economía, 2018c).

Sin embargo, en las reuniones ministeriales y del SOM durante 2019, la construcción de acuerdos se aprecia más compleja. En ese contexto, el gobierno estadounidense ha señalado que APEC debe apoyar de manera decisiva el sistema multilateral de comercio. Estas diferencias incluso fueron observadas en la Cumbre de Líderes en 2018, por lo que no se lograron consensos entre las economías miembro (por primera vez en la historia de ese foro) para la declaración final. Estados Unidos ha insistido en que para ese foro regional está fuera de sus competencias el negociar temas referentes a la OMC y debe concentrarse, más bien, en “cuestiones prácticas” (Secretaría de Economía, 2019).

Por lo anterior, la oposición de Washington rompía una práctica en la que APEC hacía eco de los esfuerzos desarrollados en la OMC para impulsar un comercio más abierto. Esto marca, de nuevo, un retorno a una posición menos protagónica de los Estados Unidos en APEC, como se observó en los primeros años de la fundación de ese foro hasta que con la administración demócrata de William Clinton se implementó una estrategia más activa que derivó en la inclusión de la agenda de liberalización económica y la formalización de la Cumbre Informal de Líderes para dotarle un respaldo político de alto nivel en las decisiones de ese foro intergubernamental. Una reflexión obligatoria sería — dentro de ese marco de baja intensidad de los Estados Unidos en los trabajos de APEC e incluso de su credibilidad¿qué posición tomará México?, ¿existirá una actitud de mayor convergencia con Washington 
a fin evitar desavenencias en materia comercial y en lo político?, ¿será una coyuntura óptima para ejercer una acción más proactiva en APEC sin mayores restricciones en tanto que APEC no es prioridad para la política comercial estadounidense?, ¿la cancelación de la reunión en Chile 2019 abrirá una ventana de oportunidad para México?

\section{MÉXICO EN LOS ESCENARIOS PROSPECTIVOS DE APEC}

La construcción de los escenarios de acción de México ante el futuro de APEC está condicionada por la imbricación de diversos factores de carácter exógeno y endógeno. Los primeros se definen por un ambiente menos favorable para el funcionamiento óptimo del sistema de comercio internacional, que se encuentra debilitado por los embates del proteccionismo y las disputas económicas, así como la actitud reticente de los Estados Unidos en dar su voto de confianza a las instituciones multilaterales, adoptando una estrategia de unilateralismo comercial. La decisión del gobierno chileno de Sebastián Piñera de cancelar, por primera vez en la historia de ese foro regional, las reuniones ministeriales y la Cumbre Informal de Líderes, más allá de los problemas sociales y políticos que orillaron a esa decisión, es claro que asesta un golpe importante a ese mecanismo de cooperación.

En el plano interno, el gobierno de López Obrador ha marcado prioridad en la no confrontación y estrechar la alianza con los Estados Unidos, aunque lo anterior implique ceder abiertamente capacidad de réplica, como se observó en las negociaciones del T-MEC y en la amenaza de la imposición de aranceles para mejorar la eficiencia en la contención de los flujos migratorios provenientes de Centroamérica. El principio de evitar "contaminar" los temas políticos con los económicos, que las administraciones anteriores habían defendido en sus relaciones con Washington, ahora no ha tenido más remedio que aceptarse ante las presiones del gobierno de Donald Trump.

Lo anterior se sumó a la incertidumbre que marcó la aprobación del T-MEC por parte del Congreso de los Estados Unidos y los escándalos políticos que involucraron al presidente Trump. El papel del ejecutivo mexicano en la política exterior ha desaparecido frente a una suerte de aislacionismo nativista (Astie-Burgos, 2019) en el que la cancillería es la encargada 
de dar respuestas de manera pragmática, perdiendo así visibilidad frente a los temas sensibles para el país y la oportunidad de ejercer liderazgo en América Latina.

Con base en lo anterior se pueden delinear los siguientes supuestos sobre el papel de México en las relaciones económicas y políticas internacionales:

a) México se concentró en la aprobación del T-MEC como un instrumento de comercio esencial en sus relaciones económicas y políticas con los Estados Unidos.

b) La política comercial externa mexicana redujo sus capacidades de acción multidireccional ante la centralidad de la agenda con los Estados Unidos.

c) La proyección de la visibilidad de México con los foros regionales y multilaterales se opaca ante la ausencia del Ejecutivo mexicano.

d) La política exterior, y en específico la de orientación económica, asume una actitud pragmática sin protagonismo.

e) México "administra" sus relaciones con el mundo sin mayor energía institucional ante las políticas de austeridad y recorte presupuestal.

Con base en los elementos anteriormente señalados, pueden considerarse dos escenarios: el posible y el deseable.

\section{El síndrome de Mexi-komori}

Una de las patologías sociales existentes en el Japón contemporáneo es la tendencia de algunos jóvenes de negarse a salir de su cuarto como una forma de expresar su rechazo en interactuar en la sociedad rígida y con estrictas reglas de comportamiento, como lo es la japonesa. Se les denomina Hikikomori (Romero, 2019). Con todas las licencias del caso, puede adaptarse esa figura para comprender el comportamiento de un Estado y de sus líderes cuando su interacción en el mundo baja de intensidad mediante la ausencia del responsable del gobierno en los foros internacionales, sacrificando visibilidad y mermando su capacidad de apuntalar los intereses nacionales en la voz del Ejecutivo.

Lo anterior se ajusta a la actitud de México en el despliegue de sus estrategias internacionales, lo que, como ya se ha afirmado, expone un repliegue combinando pragmatismo y centralidad en su agenda con los Estados Unidos. Si bien la SRE y SE 
mantienen el día a día de los contactos político-diplomáticos y económicos, ese repliegue no implica una estrategia más focalizada para un papel más asertivo en sus relaciones internacionales, sino más bien reaccionar ante los temas que se presenten en la agenda bilateral y multilateral. APEC es y será un ejemplo más.

\section{Lo anterior genera:}

1. Retroceso en las estrategias de la "diplomacia de Cumbres" ante la inasistencia del Ejecutivo mexicano en los foros internacionales, lo que genera una pérdida de oportunidad para abordar temas clave de política exterior con otros líderes mundiales.

2. El fortalecimiento de la proxy-diplomacia, es decir, el envío de un representante de “confianza” (el secretario de Relaciones Exteriores) para desarrollar el diálogo y negociación de temas de interés para el país con sus contrapartes.

3. Desarrollo de encuentros y diálogo triangular mediante la proxy-diplomacia al evitar el abordaje de temas directamente con los líderes mundiales.

4. Dar prioridad a la diplomacia telefónica, como la adoptada por el mandatario mexicano en las relaciones con el presidente Donald Trump.

Para el APEC:

a) México reduce su visibilidad, limitándose en colaborar en las acciones instrumentales del foro y profundizando su focalización y selección en los temas de la agenda.

b) Se profundiza la política de evitar "conflictos" con Washington, por lo que no habrá posicionamientos divergentes en temas sensitivos dentro de APEC a pesar de la baja prioridad que Washington tiene en el foro.

c) Hay escasa voluntad política real para contener la llamada "crisis del multilateralismo", contribuyendo indirectamente, como efecto colateral, al mayor cuestionamiento y debilitamiento de APEC.

d) La adopción de la estrategia de "hacer lo que se puede" frente al proceso de "austeridad republicana", donde la presencia de las representaciones de las dependencias mexicanas en los comités y grupos de trabajo en el APEC será más escasa, limitan la presencia de altos funcionarios mexicanos en las reuniones ministeriales y del SOM. 
e) México pierde la oportunidad de tener mayor visibilidad ante el default chileno para posicionar nuevos temas orientación social en la agenda de ese foro regional (Uscanga, 2006).

\section{"Beyond uncle Sam”}

El presente escenario parte de que, a pesar de la centralidad de la política exterior de México en las relaciones con los Estados Unidos, se implementan esfuerzos consistentes en ampliar los campos de acción geográficos de la agenda internacional del gobierno mexicano a otras regiones del mundo, más allá de América del Norte y Latinoamérica. Lo anterior implica desplegar una estrategia más integral, recuperando el discurso de la mayor visibilidad de México en lo global y la capacidad de un diálogo asertivo con sus socios estratégicos, en particular en el Pacífico asiático.

Se abandona la estrategia de encapsular la proyección de México en el exterior sin el acompañamiento del responsable del poder Ejecutivo, en la que López Obrador decide, de manera gradual, asistir a reuniones internacionales más representativas en organismos multilaterales y regionales para emprender un diálogo directo con sus contrapartes.

Lo anterior produce:

a) Posicionar temas de interés del gobierno de la "4T" (“Cuarta Transformación”) en los foros internacionales en voz del presidente de la República abandonando el "parroquialismo nativista".

b) La proxy-diplomacia baja de intensidad, canalizando las atribuciones del secretario de Relaciones Exteriores a las tareas propias de sus funciones y diálogo con otros cancilleres, siendo una excepción (más no la norma) la representación del Ejecutivo mexicano en los foros globales.

c) El establecimiento de conversaciones de alto nivel de manera directa por parte del presidente mexicano para la atención de temas sensibles de la agenda internacional del país.

d) La "diplomacia telefónica" complementa los diferentes mecanismos de diálogo de López Obrador con otros líderes mundiales, pero no sustituye los encuentros directos. 
Para el APEC:

a) México mantiene la estrategia de focalizar y seleccionar los temas dentro de la vasta agenda de APEC considerados importantes e incluso prioritarios para el proyecto de desarrollo del país, sumando nuevas áreas de visibilidad y "liderazgo con orientación a tareas" en temas sociales.

b) El desinterés de los Estados Unidos en APEC permite un margen de maniobra más amplio para emprender acciones que afecten el ambiente abierto para el libre comercio, y sumarse a las discusiones sobre la reforma institucional de APEC.

c) A pesar de los programas de austeridad, la participación mexicana en los comités y grupos de trabajo se mantiene, impulsando nuevos temas y compartiendo experiencias de las acciones del proyecto social del gobierno de López Obrador.

d) México decide organizar en 2023 o 2024 la reunión ministerial y Cumbre Informal de Líderes de APEC, presentando temáticas sobre las distorsiones sociales generadas por las estrategias de crecimiento económico.

e) México recupera visibilidad en América Latina y profundiza su liderazgo en Alianza de Pacífico, proyectando temáticas en APEC ante el "default chileno".

Los dos escenarios descritos parten de una serie de supuestos ligados al grado del avance del proyecto del gobierno de Andrés Manuel López Obrador, los márgenes de gobernabilidad y estabilidad política, así como el estado de los fundamentos macroeconómicos de la economía mexicana. En lo externo, sin duda, el T-MEC permitirá mayor certidumbre y la negociación de los temas con los Estados Unidos se mantendrá, dentro de los márgenes posibles, de manera estable, con la subsistencia de temas sensibles como la migración, la inseguridad y lucha contra el crimen organizado, cuyos disensos no ponen en riesgo el diálogo bilateral en todos los niveles.

Asimismo, ambos escenarios marcan una ruta de doble vía que pone el relieve en la capacidad de lo que México tendrá que aportar al APEC en el mediano y largo plazos, pero también qué trayectoria se va a evidenciar, y sobre todo qué clase de miembro será México dentro de ese foro intergubernamental. 


\section{REFLEXIÓN FINAL}

Después de tres decenios del surgimiento de APEC, el clima internacional ha cambiado diametralmente. El anhelo depositado en el entonces nuevo mecanismo de cooperación regional para enfrentar la amenaza del proteccionismo, ante los problemas que implicaban las conclusiones exitosas de las negociaciones de comercio multilateral, en la actualidad, por el contrario, observa el embate de las acciones proteccionistas y los neonacionalismos (de derecha e izquierda) que cuestionan la globalización y los organismos internacionales creados en la posguerra.

Como se observó, el panorama no es prometedor para APEC (y tampoco para otros espacios de diálogo regional); se requiere de la voluntad política de las economías miembro, mantener su momentum, al ser el único mecanismo de contacto a nivel ministerial y de alto nivel (sin contar la interacción que se genera en los grupos de trabajo con el sector público, privado y la academia), o el contribuir a su proceso de extinción gradual y paulatina. Ésa es la gran disyuntiva que tendrá que enfrentar en el corto y mediano plazos, en la que México debe decidir qué papel asumirá.

Las opciones son limitadas: emprender un ensimismamiento reduciendo la visibilidad del Ejecutivo mexicano o emprender una ruta de mayor proyección, transitando diversos caminos en los que la voz del presidente mexicano sume (más no reste) a la proyección más asertiva y estratégica dentro de las relaciones internacionales como una realidad cada vez más volátil y cambiante.

El falso dilema de seleccionar, como lo hace Andrés Manuel López Obrador, entre la atención de la agenda interna y los asuntos internacionales suprime la valoración de que esas dos esferas deben ser complementarias y retroalimentarse. Es justo un foro como APEC que permite - en la atención de su amplia agenda con temas transversales, y en la que se pueden realizar propuestas y acciones que puedan favorecer el interés nacional de México y, en particular, sus deseos de desarrollo - la generación de bienestar y una mejor distribución del ingreso. Lo anterior tendrá efecto siempre y cuando se apropien y se puedan alinear las recomendaciones de ese foro regional con la política pública como parte de un proyecto de nación viable y consistente. Las economías que integran a APEC (con sus diferencias en 
materia de desarrollo) aportan experiencias básicas que México no ha valorado e implementado de manera integral. Independientemente de las críticas expresadas a ese foro intergubernamental, su mayor valor quizá sea ése: ser un escaparate que permite —en un solo lugar- poner en la balanza las capacidades para emprender la ruta de un crecimiento incluyente, sustentable y sostenible, como lo ha sido el caso de la experiencia de muchas de las economías asiáticas.

\section{REFERENCIAS}

Anguiano, E. (2001). “Las relaciones México-APEC, 1995-2000”, Foro Internacional, XLI (4), 858-860.

APEC (2011). "Enhancing Small and Medium-Sized Enterprises Participation in Global Production Chains", APEC Economic Leaders' Declaration, Annex B, Honolulú, Hawái. Recuperado de https://www.apec.org/Meeting-Papers/LeadersDeclaration/2011/2011_aelm/2011_aelm_annexB

Astie-Burgos, W. (27 de septiembre de 2019). "Política exterior: Inesperado pragmatismo". Recuperado de El Universal: https://www.eluniversal.com.mx/opinion/walter-astieburgos/politica-exterior-inesperado-pragmatismo

Cárdenas, H. (1993). "Ingreso de México al mecanismo de Cooperación Económica AsiaPacífico (APEC): Nuevas implicaciones”, Comisión Mexicana de la Cuenca del Pacífico, (4)2, octubre-diciembre.

De la Mora Sánchez, L. M. (2019). "La política comercial de México durante el gobierno del presidente Enrique Peña Nieto”, Foro Internacional, LIX(3-4), 978-984.

Derbez, L. E. (2002). “México y el Mecanismo de Cooperación Asia Pacífico”, Comercio Exterior, 52(10), octubre, 872-873.

Hernández, M. C., y Martínez Jurado, J. (2019). “APEC en el radar de México”. En González García, J. (coord.), Los retos de México en el Asia Pacífico, México, Siglo XXI Editores. 
León-Manríquez, J., y Álvarez, L. F. (2020). “The Long Shadow of NAFTA. An Economic Geography of Mexico’s Integration into de Pacific Rim”. En Briceño-Ruiz J., y Lombaerde, P. de, The Political Economy of New Regionalisms in the Pacific Rim (pp. 269-129), Nueva York, Routledge.

Ojeda, R. (2000). "México y el Mecanismo de Cooperación Económica Asia-Pacífico", Revista Mexicana de Política Exterior, 61, julio-octubre, 291-293.

Romero, M. E. (2019). “Hikikomori. Las voces silenciosas de la sociedad japonesa”, México y la Cuenca del Pacífico, (8)23, mayo-agosto, 123-138.

Secofi (1997-1998). Informe de labores 1997-1998, México.

Secretaría de Economía (2003). Informe de labores, México.

Secretaría de Economía (2006). Informe de labores, México.

Secretaría de Economía (2018a). Tercera reunión de Funcionarios de Alto Nivel (SOM3) y trabajos por comités, México.

Secretaría de Economía (2018b). Libro blanco. Política Comercial Internacional, México.

Secretaría de Economía (2018c). Semana de Líderes Económicos, Foro de Cooperación Económica Asia-Pacífico (APEC), México.

Secretaría de Economía (2019). Primera reunión de Funcionarios de Alto Nivel (SOM1), Mecanismo de Cooperación Económica Asia-Pacífico (APEC), México.

Traslosheros, G. (2019). "México y el Mecanismo de Cooperación Económica Asia Pacífico (APEC)", México y la Cuenca del Pacífico, (8)23, mayo-agosto, 17-18.

Uscanga, C. (2000a). "México frente al Asia-Pacífico y sus foros regionales de cooperación". En López Villafañe, V., y Uscanga, C. (coords.), México frente a las grandes regiones del mundo (pp. 138-170), México, Siglo XXI Editores.

Uscanga, C. (2000b). "Política económica exterior de México en la región del Pacífico Asiático: estrategias después de la crisis de 1994”. En Uscanga, C. (coord.), Los nuevos desafíos de la política exterior de México en los escenarios regionales (pp. 139-152), México, Institución Paradigma de Actividades Científico Culturales S.C. 
Uscanga, C. (2004). México en los mercados regionales del Pacífico asiático, Mexico, FCPYS-UNAM.

Uscanga, C. (2006). "El movimiento anti-APEC: Escenarios de Impacto en las relaciones transpacíficas del siglo XXI”. En Sierra Kobeh, M. L., y Romero, C. A., Continuidad y cambio en los escenarios regionales. Una visión prospectiva (pp. 221-244), México, FCPyS-UNAM.

Uscanga, C. (2018). "El Acuerdo de Libre Comercio en el Asia Pacífico (ALCAP): ¿Una agenda para la política comercial de México?” En Uscanga, C., et al. (coords.), China, Japón y Corea del Sur en las estrategias económicas de México: La política comecial frente a las tendencias del neoproteccionismo de Estados Unidos (pp. 186-196), México, FCPyS-UNAM. 